\title{
An X-ray selected sample of radio-loud quasars
}

\author{
A. Wolter ${ }^{1}$ and A. Celotti ${ }^{2}$ \\ 1 Osservatorio Astronomico di Brera, via Brera 28, 20121 Milano, Italy \\ e-mail: anna@brera.mi.astro.it \\ 2 S. I. S. S. A., via Beirut 2-4, 34014 Trieste, Italy \\ e-mail: celotti@sissa.it
}

Received 29 August 2001 / Accepted 16 March 2001

\begin{abstract}
We construct the first X-ray selected sample of broad line radio-loud AGN from the EMSS survey. In order to test unifying schemes for radio-loud objects, their spectral and statistical properties (both flat and steep spectrum objects) are examined and compared with those of other samples of blazars. The X-ray selection allows us to explore properties of radio-loud quasars 10-100 weaker in the radio band than classical samples. The most convincing interpretation of our results is that there are no radio-loud quasars whose synchrotron emission peak reaches the EUV-soft X-ray band at these (radio) flux levels. Moreover, due to the comparatively weak non-thermal emission, a quasi-thermal component appears to contribute at optical-UV energies. The detection of sources at low radio fluxes also reveals a large population of steep spectrum quasars and the lack of the predicted turnover in the quasar (radio) counts. The evolution of X-ray selected radio-loud quasars does not significantly differ from that of radio-selected ones, and flat spectrum and steep spectrum sources appear to behave quite similarly.
\end{abstract}

Key words. galaxies: active - galaxies: evolution - galaxies: luminosity function - quasars: general - radio continuum: galaxies - X-rays: galaxies

\section{Introduction}

The selection energy band in the construction of complete samples constitutes one of the main biases and a source of confusion in the understanding of the Active Galactic Nuclei (AGN) phenomenology, which has often led to useful but somehow unphysical classifications.

Among radio-loud objects, a specific and instructive example - which will constitute a reference in the following - is given by the case of BL Lac objects. Samples of $\mathrm{BL}$ Lacs selected in the radio and $\mathrm{X}$-ray bands revealed systematic differences in their spectral energy distributions (SED), polarization, variability properties and evolution (e.g. Urry \& Padovani 1995). Different models have been proposed to account for this apparent "dichotomy" (Stocke et al. 1985; Ghisellini \& Maraschi 1989; Celotti et al. 1993), and only recently it has been explicitly recognized that an intrinsic continuous distribution in the position of the peak of the broad band SED of these sources might naturally lead to the detection of different types

Send offprint requests to: A. Wolter, e-mail: anna@brera.mi.astro.it of objects depending on the selection band (Padovani \& Giommi 1995). This new perspective has allowed us to have a better view of the intrinsic, physical properties of BL Lacs and speculate on their origin.

The SED of all blazars can be well represented by two main spectral components, believed to be produced as synchrotron and inverse Compton emission, respectively. One suggested possibility then is that the broad band spectral distributions of "X-ray selected" (XBL) and "radioselected" (RBL) objects are simply distinguished by the energy location of the peak of the synchrotron emission: at $\mathrm{UV} / \mathrm{X}$-ray in the former ones (High peak BL Lacs, HBL) and around the optical in the latter ones (Low peak BL Lacs, LBL; Padovani \& Giommi 1995), while the Compton peak is located in the $\gamma$-ray band (from $\mathrm{MeV}$ to $\mathrm{TeV}$ energies).

Furthermore, the entire blazar population, ranging from HBL, to LBL to Flat Spectrum Radio Quasars (FSRQ) can be interpreted, so far, as a sequence from lineless, low power sources, to broad lined, powerful quasars characterized by the (decreasing) peak energies of the two spectral components, the increasing source radio power 
and the increasing ratio between the Compton and synchrotron luminosities (Fossati et al. 1998). This trend in the SED can be physically accounted for by an increase in an external soft photon field along the sequence, in agreement with the observed behavior (Ghisellini et al. 1998). Clearly, this scenario makes definite predictions on the relationship between (radio) source power and spectral shape.

In order to understand the properties of a population it appears therefore crucial to select sources in different spectral bands, and compare their characteristics. To this aim, i.e. disentangling the intrinsic features from the selection induced ones, we have considered radio-loud quasars - which so far have been extracted from radio surveys and studied their broad band and cosmological properties when selected in $\mathrm{X}$-rays.

The sample comprises the radio-loud quasars (i.e. broad line AGN) detected in the Einstein Medium Sensitivity Survey (EMSS; Gioia et al. 1990; Stocke et al. 1991). More specifically, we have constructed the first statistically complete $\mathrm{X}$-ray selected sample of (39) radioloud quasars, in order to: a) determine whether their spectral properties differ from those of the radio-selected quasars and other classes of radio-loud objects; b) discuss the properties of these objects in the context of the beaming scenario and emission mechanisms for blazars; c) determine the statistical properties of this new sample, in the light of the unification scheme for powerful radioloud objects (e.g. Urry \& Padovani 1995 and references therein), where flat spectrum radio quasars are high power (FR II) radio galaxies in which the relativistically beamed component dominates.

With the selection criteria adopted, the sample comprises both Flat Spectrum (FS, blazar-like) and Steep Spectrum (SS) radio quasars ${ }^{1}$. It should be reminded that the radio emission in the latter ones is believed to originate from the extended radio components. In these sense the SS quasars will be not directly included in the comparison of blazar properties, but we will study and examine their optical, X-ray spectral characteristics and their statistical properties with respect to the FS quasars in this sample.

After discussing the sample selection (Sect. 2), we present the broad band spectral properties of the objects, distinguishing between flat and steep radio sources (Sect. 3) and compare them with those of other blazars (Sect. 4). The statistical and evolutionary properties of the new sample are derived in Sect. 5, while in the final Sect. 6 we discuss our results.

Hereafter we adopt cosmological parameters $H_{0}=$ $50 \mathrm{~km} \mathrm{~s}^{-1} \mathrm{Mpc}^{-1}$ and $q_{0}=0$.

\footnotetext{
1 Note that the sample is not selected on the point-like optical appearance, and thus the SS sources possibly include Broad Line Radio Galaxies.
}

\section{The sample}

The EMSS provides us with well defined X-ray selected samples of extragalactic sources. The virtually complete identification status makes it a very powerful tool to study statistical properties of classes and sub-classes of astronomical sources.

Radio information for all the (extragalactic) EMSS objects is available from VLA snapshots at $6 \mathrm{~cm}$ taken during the identification process (e.g. Maccacaro et al. 1994), with a flux limit of about $1 \mathrm{mJy}$, corresponding to 5 times the rms of the observations. Since we intend to compute the radio spectrum using the recently released data at $20 \mathrm{~cm}$ from the NRAO VLA Sky Survey (NVSS; Condon et al. 1998), we cut the EMSS sample at $\delta \geq-40^{\circ}$. With this cut, that excludes only $5 \%$ of all the AGNs and only one radio-loud, the EMSS contains 79 radio detected AGN, of which 43 have a two-point spectral index ${ }^{2}$ between radio and optical $\left(\alpha_{\text {ro }}\right)$ larger than 0.35 (Della Ceca et al. 1994) and are therefore classified as radio-loud. We exclude another four objects for the reasons detailed in the Appendix, thus constructing a complete sample of 39 radio-loud quasars, that we dub the EMSS Radio-Loud quasar sample (ERL).

In the following we will refer to FS and SS spectrum radio-loud objects, where the division is formally considered to be at $\alpha_{\mathrm{r}}=0.7$ (see e.g. Perlman et al. 1998) which leads to a similar statistics for the two sub-classes (20 FS and $19 \mathrm{SS}$ ). As the distribution of $\alpha_{\mathrm{r}}$ does not show any suggestion of being the sum of two different (bimodal) distributions (see Fig. 1), the choice of a dividing value is somewhat arbitrary. In any case, the results of this work are not affected by a different choice (the case $\alpha_{\mathrm{r}}=0.5$ has been also explored).

The list of sources and information is presented in Table 1. Columns are as follows: (1) Name of the source in the EMSS catalog - numbers refer to notes to the table, indicating either other names from literature or the source radio morphology; (2) Redshift; (3) $5 \mathrm{GHz}$ flux from EMSS, unless otherwise stated, in mJy; (4) $1.4 \mathrm{GHz}$ flux from NVSS; (5) Radio spectral index; (6) Magnitude, from EMSS; (7) unabsorbed X-ray flux (0.3-3.5 keV band), from EMSS; (8) Galactic $N_{\mathrm{H}}$, from Stark et al. (1992); (9) X-ray spectral index from EMSS data; (10) X-ray spectral index from ROSAT data (Brinkmann et al. 1997); (11-13) $\alpha_{\mathrm{ro}}, \alpha_{\mathrm{ox}}$ and $\alpha_{\mathrm{rx}}$.

The distributions of radio spectral indices and luminosities in the three bands are plotted in Fig. 1 for FS and SS, respectively.

\footnotetext{
${ }^{2}$ While the original EMSS two point spectral indices $\alpha_{\text {ro }}$, $\alpha_{\mathrm{ox}}, \alpha_{\mathrm{rx}}$ were estimated at $5 \mathrm{GHz}, 2500 \AA$ and $2 \mathrm{keV}$, comoving energies, here we recompute them at $5 \mathrm{GHz}, 5500 \AA$ and $1 \mathrm{keV}$, comoving energies, in order to compare with values for other samples. For the K-correction in the optical and X-ray bands it is assumed $\alpha_{\mathrm{o}}=\alpha_{\mathrm{x}}=1$ (see e.g. Della Ceca et al. 1994), where the spectral indices $\alpha$ are defined by $F_{\nu} \sim \nu^{-\alpha}$.
} 


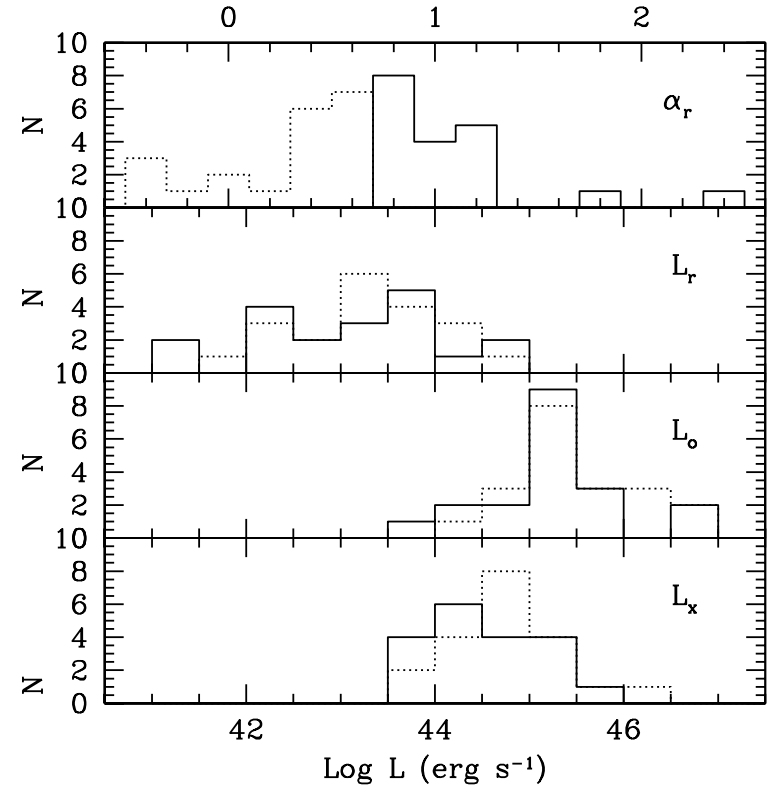

Fig. 1. Distributions of radio spectral indices and luminosities for the ERL. The FS and SS subsamples are indicated by the dotted and continuous line, respectively. The axis on top of the figure refers to the spectral index $\alpha_{\mathrm{r}}$

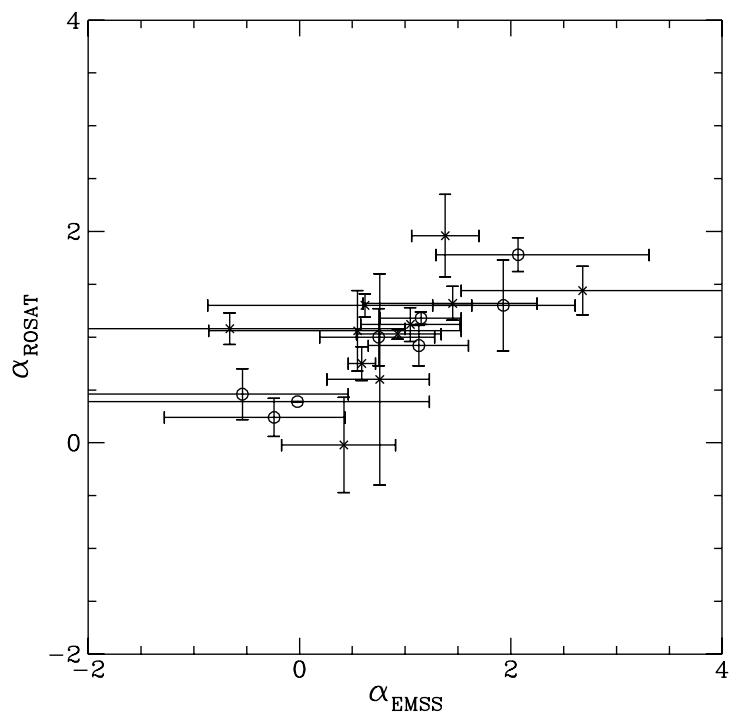

Fig. 2. The ROSAT spectral index (from Brinkmann et al. 1997) vs. the EMSS spectral index derived from the Hardness Ratio (from Maccacaro et al. 1988); FS and SS sources are indicated as crosses and circles, respectively

\section{Spectral properties}

\subsection{X-ray spectral indices}

The $\mathrm{X}$-ray spectral indices for the sample have been estimated from the IPC hardness ratios (see e.g. Maccacaro et al. 1988), assuming a power law spectral shape and Galactic line of sight absorbing columns, and compared with those determined by ROSAT. The former ones span a large interval $-2.2<\alpha_{\mathrm{x}}<2.4$, and within the uncertainties significantly correlate with the ROSAT values (see Fig. 2). Unfortunately the large errors do not allow either to detect any difference between FS and SS sources, or to infer the origin of the $\mathrm{X}$-ray component (e.g. steep synchrotron dominated versus flat Compton dominated emission).

No relationship has been found between the X-ray and radio spectral indices, the source fluxes or redshifts.

\subsection{Broad band properties}

In order to quantify the total power and SED properties of the sources and compare them with those of other samples, we compute the luminosities in the radio, optical and X-ray bands $\left(L_{\mathrm{r}}, L_{\mathrm{o}}, L_{\mathrm{x}}\right)$ and the corresponding broad band spectral indices $\left(\alpha_{\mathrm{ro}}, \alpha_{\mathrm{ox}}, \alpha_{\mathrm{rx}}\right)$.

Significant linear correlations are found between the luminosities in the three bands. However, since a possible culprit for this kind of correlation is the common redshift dependence, we test its influence by using a partial correlation analysis (e.g. Kendall \& Stuart 1979). The partial correlation coefficients are defined as:

$r_{12 \_3}=\frac{\left(r_{12}-r_{13} \times r_{23}\right)}{\sqrt{\left(1-r_{13}^{2}\right) \times\left(1-r_{23}^{2}\right)}}$,

where $r_{12}, r_{13}$, and $r_{23}$ are the Spearman rank test coefficients for two variables at a time. By assigning to index "3" the redshift variable and interchanging $L_{\mathrm{x}}, L_{\mathrm{r}}$ and $L_{\mathrm{o}}$ as variables " 1 " and " 2 " we obtain the following results: $r_{\mathrm{rx}_{-} z}=0.441 ; r_{\mathrm{ox}_{-} z}=0.325 ; r_{\mathrm{ro}_{-} z}=0.381$. The null hypothesis is rejected at the $95 \%$ level for the last two occurrences, while the radio and X-ray luminosity seem to be correlated at the $99 \%$ level.

No differences (according to the Kolmogorov-Smirnoff test) are found between the distributions relative to FS and SS, neither in luminosities (probability $p \geq 20 \%$; see Fig. 1) nor in broad band spectral indices ( $p \geq 47 \%$; see Fig. 3) nor in the trends, suggesting a behavior of the compact beamed component independent of the large scale radio flux dominance.

The shape of the SED appears however to be clearly related to the radio luminosity: in fact, the only statistically significant trends found are of both $\alpha_{\text {ro }}$ and $\alpha_{\text {rx }}$ with $L_{\mathrm{r}}$ (see Fig. 3). Although the correlation might be due to the common dependence of the two spectral indices on $L_{\mathrm{r}}$ itself, its relevance is supported by two facts: a) $L_{\mathrm{r}}$ does not correlate with $\alpha_{\text {ox }}$; b) $L_{\mathrm{r}}$ spans the largest interval in luminosity (about five decades compared to the three covered by $L_{\mathrm{o}}$ and $L_{\mathrm{x}}$ ). In any case, the presence of such a trend is indicative of the fact that through $\mathrm{X}$-ray selection we detect objects with a rather limited range in optical luminosity but a large span in the radio one, extending the range of sampled $L_{\mathrm{r}}$ towards faint sources compared to radio-selected quasars (see Sect. 4).

On the other hand, it is interesting to consider the relative behavior of the broad band spectral indices, as shown in Fig. 4. $\alpha_{\mathrm{rx}}$, which spans the smallest interval, and $\alpha_{\mathrm{ox}}$, which spans the largest one, do not show a strong correlation (98\% level, according to the Student's-t test), 
Table 1. The sample

\begin{tabular}{|c|c|c|c|c|c|c|c|c|c|c|c|c|}
\hline Name & $z$ & $F_{5}^{\mathrm{a}}$ & $F_{1.4}^{\mathrm{b}}$ & $\alpha_{\mathrm{r}}$ & mag & $F_{\mathrm{X}}^{\mathrm{c}}$ & $N_{\mathrm{H}}^{\mathrm{d}}$ & $\alpha_{\mathrm{X}}$ & $\mathrm{AT}$ & $\alpha_{\mathrm{ro}}$ & $\alpha_{\mathrm{ox}}$ & $\alpha_{\mathrm{rx}}$ \\
\hline MS 0012.5-0024 & 695 & 24.0 & 14.9 & 0.40 & 8.51 & 2.41 & 2.9 & $1.50_{-0.63}^{+0.70}$ & & 0.32 & 1.33 & 0.67 \\
\hline $0038.8-0159^{1}$ & 675 & 322.0 & 34.6 & 0.97 & 6.86 & 3.07 & 2.9 & $-0.31_{-1.17}^{+0.69}$ & & .53 & 1.54 & 0.88 \\
\hline 2 & 88 & 5.6 & 7.0 & 0.19 & .00 & 1.48 & 2.9 & - & & .30 & 1.33 & 0.66 \\
\hline MS 0 & 308 & 27.6 & 40.6 & 0.32 & 18.44 & 3.33 & 3.7 & - & & 0.42 & 1.29 & 0.72 \\
\hline MS 0136.3+0606 & 0.450 & 16.3 & 67.5 & 1.18 & 18.52 & 2.72 & 3.7 & $2.07_{-0.78}^{+1.24}$ & 1 . & 0.41 & 1.30 & 0.72 \\
\hline MS 0152.4+0424 & 1.132 & 184.1 & 323.8 & 0.47 & 19.17 & 6.28 & 4.2 & $0.50_{-0.63}^{+0.50}$ & & 0.63 & 1.07 & 0.78 \\
\hline MS 0225.5-1052 & 1.038 & 60.0 & 132.2 & 0.66 & 19.34 & 1.56 & 2.5 & $-0.66_{-\infty}^{+1.66}$ & $1.08 \pm 0.15$ & 0.56 & 1.29 & 0.81 \\
\hline MS $0226.8-1041^{2}$ & 0.620 & 96.2 & 293.2 & 0.93 & 18.32 & 2.53 & 2.5 & $1.13_{-0.48}^{+0.47}$ & $0.92 \pm 0.19$ & 0.54 & 1.35 & 0.82 \\
\hline MS $0232.5-0414^{3}$ & 1.450 & 522.9 & 1495.9 & 0.87 & 16.28 & 7.19 & 2.5 & $1.15_{-0.39}^{+0.38}$ & $1.18 \pm 0.06$ & 0.51 & 1.49 & 0.85 \\
\hline MS 0311.8-0801 & 1.250 & 8.2 & 70.3 & 1.78 & 19.12 & 3.38 & 5.4 & $1.10_{-1.19}^{+0.92}$ & & 0.44 & 1.17 & 0.70 \\
\hline MS $0402.0-3613^{4}$ & 1.417 & 2019.0 & 1132.3 & -0.48 & 17.17 & 33.98 & 0.8 & $0.59_{-0.13}^{+0.13}$ & $0.75 \pm 0.16$ & 0.59 & 1.12 & 0.78 \\
\hline MS 0438.6-1050 & 0.868 & 26.8 & 51.2 & 0.54 & 18.97 & 3.48 & 5.9 & $-1.45_{-\infty}^{+1.90}$ & & 0.46 & 1.19 & 0.71 \\
\hline MS $0521.7+7918$ & 0.503 & 22.1 & 102.6 & 1.28 & 17.45 & 5.52 & 7.9 & $0.98_{-2.20}^{+1.41}$ & & 0.35 & 1.33 & 0.69 \\
\hline $98.0+4840$ & 0.700 & 75.0 & 160.3 & 0.63 & 17.96 & 2.97 & 4.6 & $1.45_{-0.85}^{+0.80}$ & $1.32 \pm$ & 0.48 & 1.36 & 0.78 \\
\hline MS $0815.7+5233^{5}$ & 0.624 & 1.5 & 3.6 & 0.73 & 20.75 & 3.15 & 4.3 & $2.03_{-0.72}^{+0.93}$ & & 0.37 & 0.94 & 0.56 \\
\hline MS 0822.0+0309 & 0.577 & 31.5 & 85.3 & 0.83 & 17.94 & 1.46 & 3.5 & $2.85_{-1.78}^{+9.99}$ & & 0.41 & 1.49 & 0.78 \\
\hline MS 0828.7+6601 & 0.329 & 98.8 & 235.3 & 0.72 & 20.21 & 4.90 & 4.2 & $-0.08_{-0.71}^{+0.53}$ & & 0.69 & 0.95 & 0.78 \\
\hline $3+6$ & 1.112 & $369.0^{\mathrm{e}}$ & 1275.7 & 1.03 & 18.16 & 3.84 & 4.4 & $-0.54_{-2.33}^{+1.00}$ & $0.46 \pm 0.24$ & 0.65 & 1.29 & 0.87 \\
\hline $.0+2845$ & 1.273 & 336.0 & 232.6 & -0.31 & 19.09 & 1.73 & 3.2 & - & & 0.62 & 1.29 & 0.85 \\
\hline $2+2825$ & 0.922 & 37.0 & 68.0 & 0.51 & 18.27 & 1.37 & 3.2 & $-2.48_{-\infty}^{+2.33}$ & & 0.42 & 1.46 & 0.78 \\
\hline $8.4+6913^{7}$ & 0.928 & 23.0 & 59.8 & 0.79 & 19.08 & 2.68 & 3.7 & $-0.24_{-1.04}^{+0.67}$ & $0.24 \pm 0.18$ & 0.47 & 1.22 & 0.73 \\
\hline MS $1003.6+1300^{2,8}$ & 0.648 & 125.0 & 305.7 & 4 & 5 & 2.75 & 3.8 & $1.38_{-0.70}^{+0.68}$ & & 0.49 & 1.46 & 0.82 \\
\hline MS $1050.9+5418$ & 0.995 & 167.1 & 266.0 & 0.39 & 19.25 & 4.89 & 0.8 & $0.42_{-0.59}^{+0.49}$ & $-0.02 \pm 0.45$ & 0.63 & 1.12 & 0.79 \\
\hline MS $1138.6+6553^{2}$ & 0.805 & 132.8 & 506.4 & 1.11 & 18.30 & 1.26 & 1.0 & $0.75_{-0.56}^{+0.53}$ & $1.00 \pm 0.27$ & 0.57 & 1.48 & 0.89 \\
\hline MS $1234.9+6651^{2}$ & 0.860 & 142.2 & 335.2 & 0.71 & 17.99 & 5.39 & 1.9 & $-1.21_{-\infty}^{+1.71}$ & & 0.53 & 1.29 & 0.79 \\
\hline MS $1311.1+3210^{9}$ & 0.303 & 7.0 & 132.0 & 2.44 & 18.86 & 3.29 & 1.1 & $-0.31_{-\infty}^{+1.09}$ & & 0.39 & 1.24 & 0.69 \\
\hline MS $1326.6+2546^{2,9}$ & 0.986 & 45.6 & 89.3 & 0.56 & 17.80 & 2.40 & 1.1 & $0.76_{-0.50}^{+0.47}$ & $0.60 \pm 1.00$ & 0.41 & 1.45 & 0.77 \\
\hline MS $1340.7+2859$ & 0.905 & 250.0 & 252.1 & 0.01 & 17.05 & 4.88 & 1.2 & $0.55_{-1.41}^{+0.98}$ & $1.06 \pm 0.38$ & 0.47 & 1.44 & 0.80 \\
\hline MS $1442.8+6344$ & 1.380 & 445.0 & 689.6 & 0.36 & 17.19 & 2.93 & 1.7 & $1.05_{-0.47}^{+0.47}$ & $1.12 \pm 0.16$ & 0.53 & 1.51 & 0.87 \\
\hline MS $1623.4+2712^{10}$ & 0.525 & $240.0^{\mathrm{f}}$ & 531.0 & 0.66 & 18.41 & 7.78 & 3.4 & $1.38_{-0.32}^{+0.32}$ & $1.96 \pm 0.39$ & 0.62 & 1.16 & 0.80 \\
\hline MS $1640.0+3940$ & 0.540 & 31.0 & 54.4 & 0.47 & 18.25 & 4.39 & 1.0 & $0.93_{-0.39}^{+0.41}$ & $1.03 \pm 0.05$ & 0.41 & 1.29 & 0.72 \\
\hline MS 1657.1+3524 & 0.949 & 5.6 & 5.2 & -0.06 & 20.24 & 1.11 & 1.7 & $0.62_{-1.49}^{+1.01}$ & $1.30 \pm 0.11$ & 0.39 & 1.21 & 0.67 \\
\hline MS $1837.8+45$ & 0.958 & 210.0 & 801.4 & 1.11 & 19.38 & 3.60 & 5.9 & $0.84_{-\infty}^{+1.79}$ & & 0.70 & 1.12 & 0.84 \\
\hline MS 2037.3-0035 & 0.609 & 23.1 & 73.1 & 0.96 & 18.39 & 2.21 & 6.6 & $1.93_{-0.67}^{+0.68}$ & $1.30 \pm 0.43$ & 0.42 & 1.34 & 0.74 \\
\hline MS $2113.8+0455$ & 1.001 & 122.0 & 316.8 & 0.79 & 20.37 & 2.53 & 6.5 & $-0.02_{-3.03}^{+1.25}$ & $0.39 \pm 0.00$ & 0.71 & 1.02 & 0.82 \\
\hline MS $2141.2+1730^{12}$ & 0.213 & 417.0 & 651.8 & 0.37 & 15.85 & 8.94 & 8.0 & $2.68_{-1.15}^{+1.52}$ & $1.44 \pm 0.23$ & 0.46 & 1.49 & 0.81 \\
\hline MS $2143.2+1429$ & 1.387 & 50.3 & 226.2 & 1.25 & 20.40 & 1.91 & 7.0 & - & & 0.67 & 1.06 & 0.80 \\
\hline MS 2247.8-0703 & 1.710 & 44.7 & 82.4 & 0.51 & 20.82 & 3.82 & 3.6 & $1.26_{-0.62}^{+0.59}$ & & 0.63 & 0.90 & 0.72 \\
\hline MS $2329.3-3827^{13}$ & 1.195 & 670.0 & 543.6 & -0.17 & 16.20 & 3.08 & 1.5 & $-1.05_{-\infty}^{+2.02}$ & & 0.46 & 1.65 & 0.87 \\
\hline
\end{tabular}

${ }^{\mathrm{a}}$ Flux @ $5 \mathrm{GHz}$ in mJy; ${ }^{\mathrm{b}}$ Flux @ $1.4 \mathrm{GHz}$ in mJy; ${ }^{\mathrm{c}}$ Unabsorbed flux in $(0.3-3.5 \mathrm{keV})$ in $10^{-13} \mathrm{erg} \mathrm{cm}^{-2} \mathrm{~s}^{-1}$; ${ }^{\mathrm{d}} \mathrm{Hydrogen}$ column density in $10^{20} \mathrm{~cm}^{-2}$; ${ }^{\text {e }}$ from Gregory \& Condon (1991); ${ }^{\mathrm{f}}$ from Becker et al. (1991). Notes: (1) 4C 02.04 (2) Triple radio source (3) PKS 0232-04, double radio source (4) PKS 0402-362 (5) Weak-lined, see text (6) 3C 204 (7) Radio core+extended (8) WE 1138+65 (9) WAT (10) B2.2 1623+27A (11) OU+462 (12) OX 169 (13) PKS 2329-384, HB.

while (a part for a conspicuous object, the weak lined MS 0815.7+5233 - see also Sect. 4) $\alpha_{\text {ro }}$ decreases for increasing $\alpha_{\text {ox }}$ (at $>99 \%$ level). Interestingly, this trend is qualitatively consistent with a change in the frequency position of the peak of the synchrotron spectral component.
Furthermore, by comparing Figs. 3 and 4, it is clear that this spectral behavior is connected with the source radio luminosity, as the flatter is $\alpha_{\mathrm{ro}}$, the lower is $L_{\mathrm{r}}$. Notice also that the trend does not simply relate to the optical and 


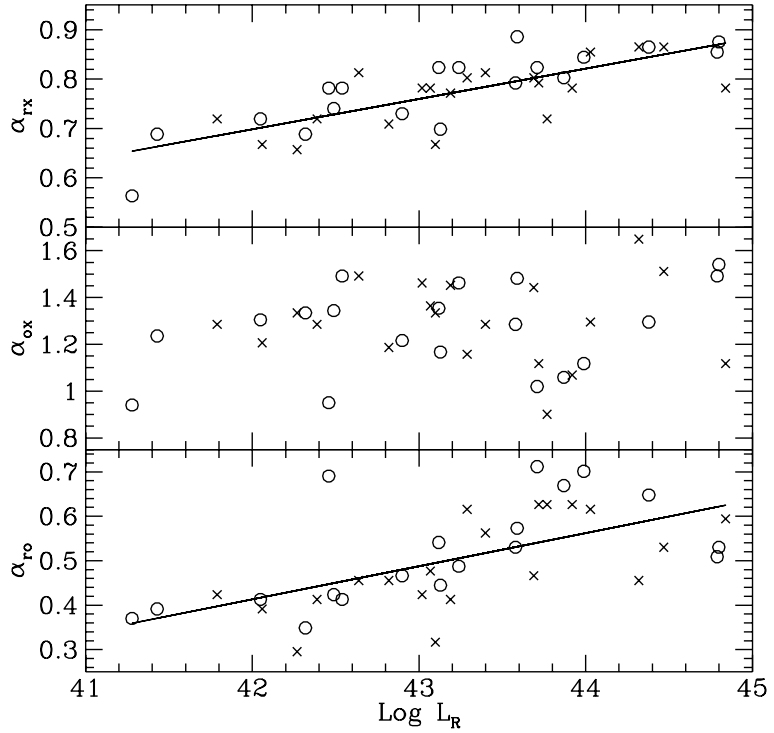

Fig. 3. Distribution of $\alpha_{\mathrm{rx}}, \alpha_{\mathrm{ox}}, \alpha_{\mathrm{ro}}$ as a function of $L_{\mathrm{r}}$. SS are represented by circles and FS by crosses. The linear regression for the entire sample is plotted for $\alpha_{\mathrm{rx}}$ and $\alpha_{\mathrm{ro}}$ (the separate regressions for FS and SS are consistent with the total), while $\alpha_{\mathrm{ox}}$ vs. $L_{\mathrm{r}}$ is not statistically significant

$\mathrm{X}$-ray luminosities, which therefore appear to be worse tracers of systematic behaviors in the SED shape.

We conclude that: a) the ERL sample (and the FS and SS subsamples) significantly extends the radio luminosity range (but not the optical one) of radio-loud quasars; b) the broad band spectral indices behavior is consistent with being caused by a change in the synchrotron peak energy; c) the trend in the SED shape correlates with the radio luminosity.

\section{Comparison with other samples}

We now compare the spectral properties of the ERL sample with those of radio-selected quasars (FSRQ and Steep Spectrum radio-loud quasars, SSQ) and (radio and $\mathrm{X}$-ray selected) BL Lac objects and examine our findings in the light of the blazar spectral sequence scenario already described and the unification of powerful radio sources. Does the $\mathrm{X}$-ray selection allow us to discover powerful lined sources whose synchrotron emission peaks at high (UV-X-rays) energies, analogously to $\mathrm{X}$-ray selected BL Lac objects? Does the detection of ERL force us to reconsider the above scenario? Do the nuclear properties of steep spectrum objects differ from those of the flat spectrum sources or their nuclear properties are independent of the extended emission?

Let us first examine the former issue.

The plane $\alpha_{\text {ox }}$ versus $\alpha_{\text {ro }}$ has been often used to characterize the SED of the above classes of sources. As mentioned in the introduction, the position of the different objects in this diagram can be accounted for by the different location of the synchrotron peak in their energy distribution.

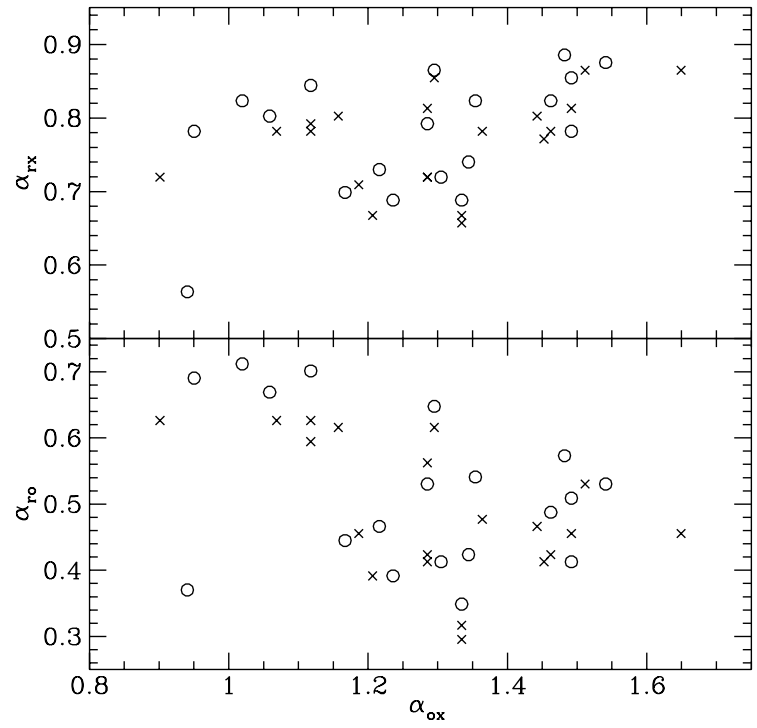

Fig. 4. Broad band spectral indices: $\alpha_{\mathrm{rx}}$ (upper panel) and $\alpha_{\mathrm{ro}}$ (lower panel) vs. $\alpha_{\text {ox }}$ for the ERL. Symbols like in Fig. 3

In Fig. 5a we show the ERL and other samples (see the figure caption) of both FSRQ and radio and X-ray selected BL Lacs. For clarity, we also plot in Fig. 5b a schematic representation of the loci occupied by the different samples, in which the mean and the $1 \sigma$ width of the distribution in the two variables are plotted. The ERL occupy the transition region (in the "boomerang" shaped blazar distribution) between FSRQ and X-ray selected ( HBL) BL Lac objects, roughly overlapping with radioselected $(\sim \mathrm{LBL})$ ones. Note that the location of LBL and FSRQ in the spectral index plane is due to the dominance in the $\mathrm{X}$-ray band of a flat Compton component.

Within the spread of the above quantities, this indicates that even $\mathrm{X}$-ray selection is unable to detect radioloud broad-lined objects with high peaked synchrotron components. Intriguingly, the object in the left-bottom corner, in the "HBL region", is the already mentioned MS 0815.7+5233, a weak lined AGN, BL Lac-like (see Stocke et al. 1991, Tables 11 and 13 and notes therein).

Furthermore, the broad band spectral indices $\alpha_{\mathrm{rx}}$ and $\alpha_{\text {ro }}$ of the ERL tend to be lower $(\Delta \alpha \sim 0.1-0.2)$ than those of the FSRQ, with the flattest sources coinciding with the very same objects which have lower $L_{\mathrm{r}}$. In other words ERL resemble radio selected quasars in the optical and $\mathrm{X}$-ray bands, while are comparatively weaker in the radio one, indeed suggesting a larger contribution (peak) to the SED at higher energies. This result is fully consistent with the high energy/X-ray selection of the sample.

It is also interesting to explore the relationship among the luminosities of sources belonging to different samples, as the lack of ERL with SED resembling those of HBL might be due, within the above scenario, to their relatively higher luminosity. In Fig. 6 the above samples are plotted in the $L_{\mathrm{x}}$ vs. $L_{\mathrm{r}}$ plane (luminosities uncorrected for evolution). While in the $\mathrm{X}$-ray band the ERL are on 
average a factor of $\sim 10$ weaker than the FSRQ and similarly the SSQ, in the radio (core) luminosities they are of the order of 100 (10) times weaker than the FSRQ (SSQ) (e.g. Fossati et al. 1998; Maraschi \& Rovetti 1994). The comparison also shows that the $L_{\mathrm{x}}$ of ERL covers the same range spun by HBL, although it extends at even higher $L_{\mathrm{x}}$. Nevertheless there is no indication of a trend for the weakest ERL to have SEDs more resembling highly peaked sources, as their radio luminosity range is above that of HBL. We stress that in the very same EMSS sample HBL are indeed found in large number (Morris et al. 1991; Wolter et al. 1991), excluding observational biases against detection of ERL peaking in the X-ray band.

These findings are therefore in global agreement with the expectation of the blazars sequence scenario described in the introduction, thus re-enforcing the view of a strong connection between the spectral energy distribution and the radio luminosity and suggesting again that the latter might be a good indicator of the source power.

However, it should be noted that ERL and LBL span a similar range of three decades in $L_{\mathrm{r}}$. While one would expect some overlapping along the sequence - as also suggested by the existence of sources changing from being BL Lac-like to quasar-like and vice versa, and RBL with FR II extended morphology - the extent of the common luminosity range seems to suggest more of a parallel behavior, rather than a continuous trend, of lined and lineless sources. However, it appears that at lower $L_{\mathrm{x}}$ the ERL might be filling the intermediate region between LBL and HBL. These two facts, and thus the overall distribution of radio and X-ray luminosities of ERL, might be ascribed to the increasing dominance of a quasi-thermal optically-UV component in quasars of increasingly lower (non-thermal) radio power. Indeed the typical $\alpha_{\text {ro }}$ and $\alpha_{\text {ox }}$ of the low radio power ERL (see Fig. 3) corresponds to SED whose peak is located in the optical-UV region. The presence of a quasi-thermal component would indeed flatten $\alpha_{\text {ro }}$ and steepen $\alpha_{\mathrm{rx}}$ in sources with decreasing non-thermal continuum. This "excess" contribution could be identified with both/either "blue bump" emission in quasars (FS) and/or contamination from the host galaxy in SS (possibly Broad Line radio galaxies).

\section{Statistical and evolutionary properties}

Let us now examine the statistical properties of the sample within the framework of the unification scheme for powerful radio sources. More specifically, do the steep radio sources evolve consistently with the postulate of the unification scheme (i.e. similarly to flat sources)?

\subsection{Number counts}

The number counts of radio-loud AGN of the EMSS have been presented by Della Ceca et al. (1994). Here we consider any possible distinction related to the radio spectral index (FS vs. SS), and compare the ERL counts to those of other samples of radio-loud objects.

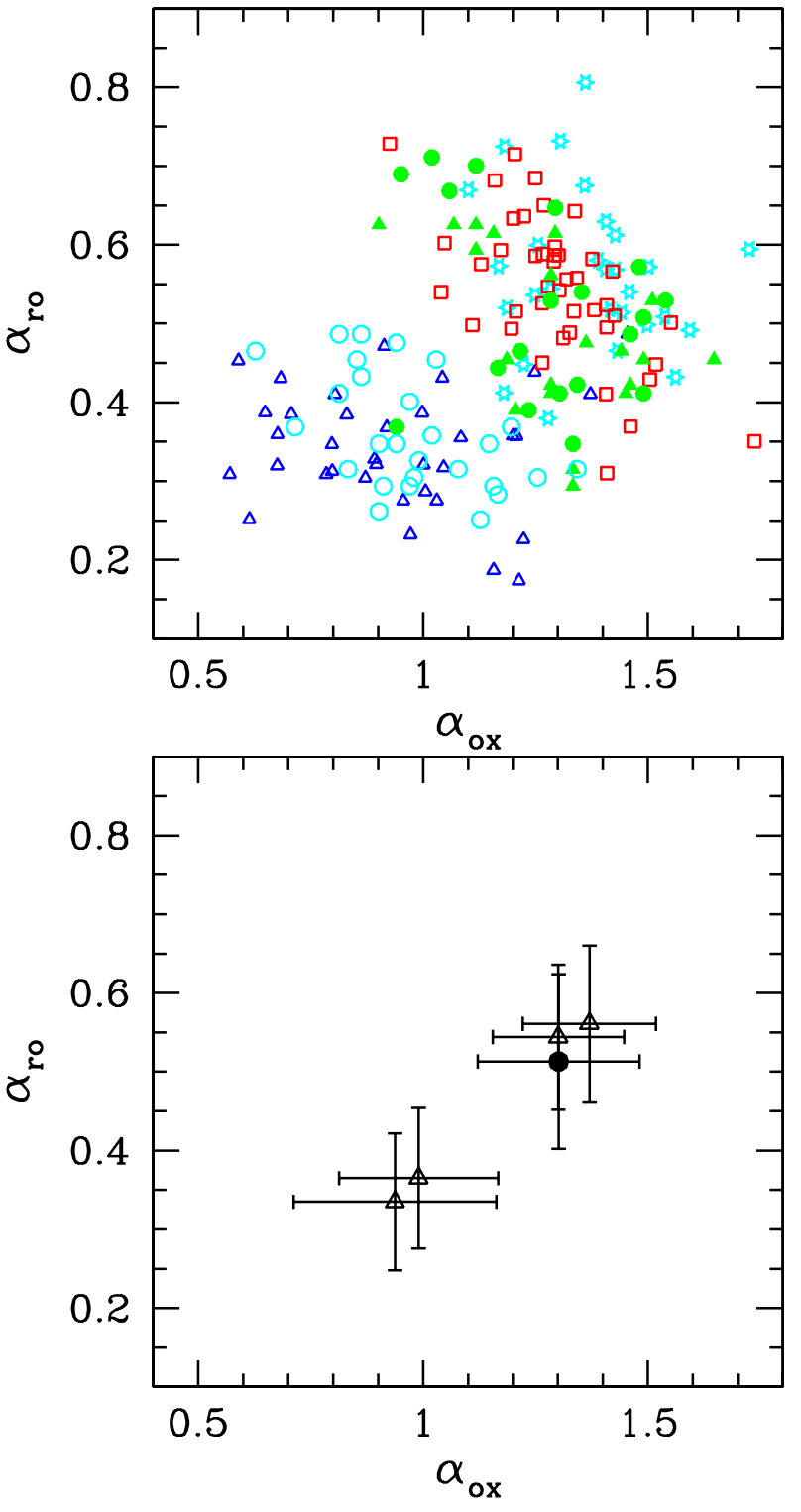

Fig. 5. a) Broad band spectral index $\alpha_{\text {ro }}$ vs. $\alpha_{\text {ox }}$ for the ERL (filled triangles and circles for FS and SS, respectively) compared to: EMSS BL Lacs (empty circles), Slew BL Lacs (empty triangles), 1 Jy BL Lacs (empty stars), 2 Jy FSRQ (empty squares). Just for graphical purposes, we have not included in the plot the most recent blazars selected by Perlman et al. (1998) and Laurent-Muehleisen et al. (1999). b) The lower panel shows the loci of the different samples, the black circle indicates the ERL sample, while the triangles the BL Lac and FSRQ samples. The errorbars represent the width $(1 \sigma)$ of the distributions in $\alpha_{\text {ro }}$ and $\alpha_{\text {ox }}$ respectively

The (integral) $\log N-\log S$ distributions for FS, SS, all ERL and BL Lacs are shown in Fig. 7, and the parametric results derived from their linear fits are reported in Table 2. (The fit to the counts is done by using the individual points in a differential form, see e.g. Gioia et al. 1991 for a description of the procedure.)

The first point to be noticed is the marginal (though it extends over the whole range in flux) evidence for flatter counts of FS, which dominate at the highest flux levels. 


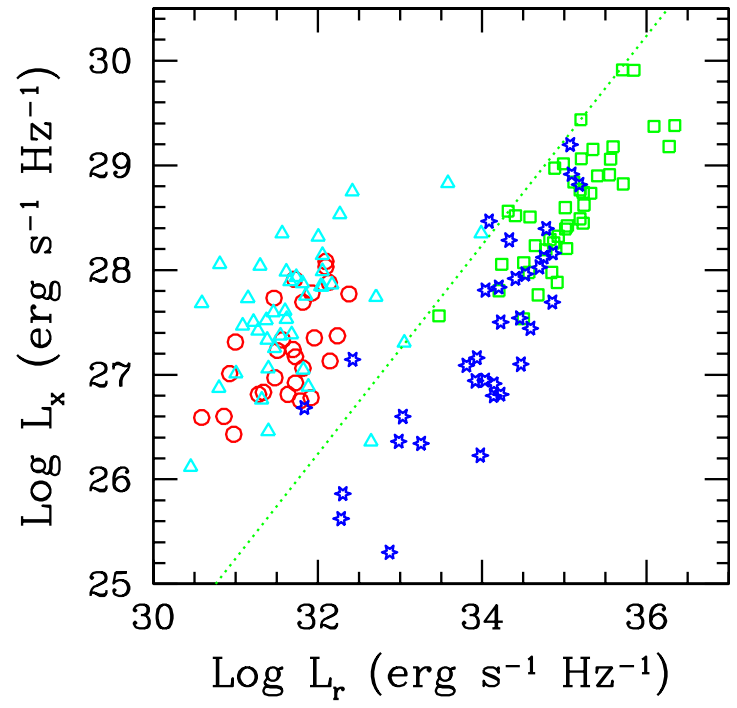

Fig. 6. Monochromatic (@1 keV) X-ray vs. monochromatic (@5 GHz) radio luminosity of the ERL, EMSS BL Lacs, Slew BL Lacs, 1 Jy BL Lacs, 2 Jy FSRQ (symbols as in Fig. 5). The dotted line indicates the "nominal separation" between HBL and LBL in the luminosity plane (as defined in Fossati et al. 1998)

Table 2. Statistical properties

\begin{tabular}{|lccc|}
\hline & ERL & FS & SS \\
\hline Count slope $^{a}$ & $1.9[1.7-2.2]$ & $1.8[1.6-2.1]$ & $2.0[1.7-2.3]$ \\
$\left\langle z^{b}\right\rangle$ & $0.92 \pm 0.40$ & $0.99 \pm 0.40$ & $0.85 \pm 0.39$ \\
$V_{\mathrm{e}} / V_{\mathrm{a}}$ & $0.78 \pm 0.05$ & $0.76 \pm 0.06$ & $0.81 \pm 0.07$ \\
$C$ & & $6.6[5.6-7.4]$ & $6.4[5.6-7.1]$ \\
$\gamma$ & & $2.9[2.5-3.1]$ & $3.0[2.7-3.2]$ \\
$\mathrm{LF}^{a}$ & $1.6[1.4-1.7]$ & $1.6[1.3-1.8]$ & $1.9[1.6-2.2]$ \\
$\log K^{c}$ & -8.74 & -8.95 & -9.00 \\
\hline
\end{tabular}

In square brackets the $1 \sigma$ confidence range.

${ }^{a}$ Power-law index of a linear fit (integral slope).

${ }^{\mathrm{b}} \pm 1 \sigma$ values.

${ }^{\text {c }} K$ is in units of $\mathrm{Mpc}^{-3} L_{44}^{\mathrm{LF}-1}$, where $L_{44}$ is the luminosity in units of $10^{44} \mathrm{erg} \mathrm{s}^{-1}$.

The count distributions of FS is in complete agreement with what found for all AGN - mostly radio quiet- in the EMSS (1.61 \pm 0.06 , Della Ceca et al. 1992), while the slopes of SS and all ERL are marginally steeper (but only at $1 \sigma$ level) than those of all AGN. Clearly the statistics does not allow us to perform more complex fits, although a single power law might be inadequate to represent the distributions.

How do the ERL counts compare with those of FSRQ and SSQ? Padovani \& Urry (1992, hereafter PU92) examined the statistical properties of the best defined radio sample, namely the 2 Jy (Wall \& Peacock 1985). This comprises $34 \mathrm{SSQ}$ and $50 \mathrm{FSRQ}^{3}$. The (poorly defined) count distribution in the radio band is consistent with

\footnotetext{
${ }^{3}$ We note here that the average radio spectral indices of the two subsamples of FS and SS are fully consistent with those of FSRQ and SSQ once the same definition is adopted (more
}

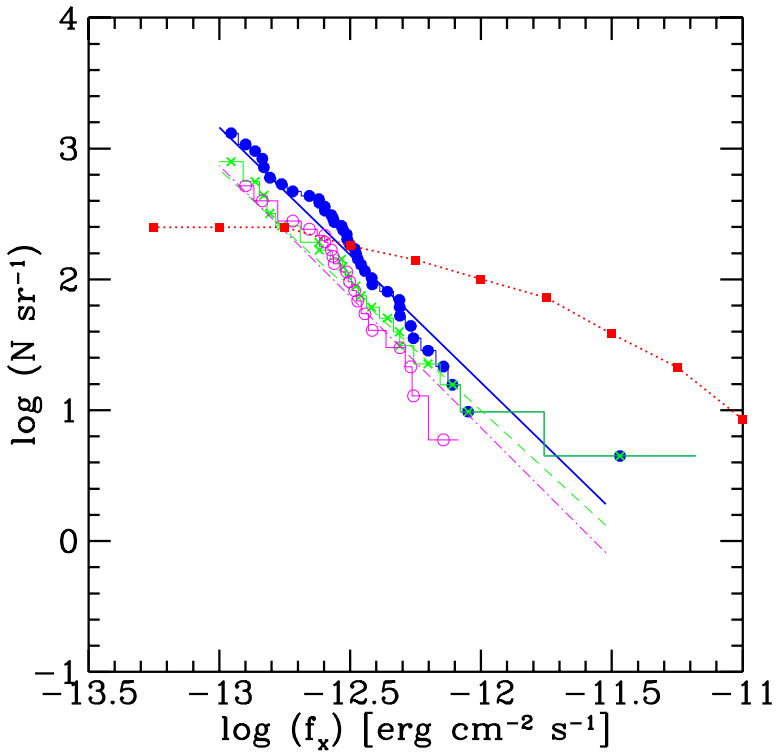

Fig. 7. Integral number counts for FS (crosses), SS (circles) and all ERL (filled circles). The lines represent the corresponding linear fits: heavy line for ERL, dashed line for FS, dotdashed line for SS. The flatter distribution (squares) represents the EMSS BL Lacs (from Wolter et al. 1991). Results of the fits are reported in Table 2

being Euclidean and also with those of FS and SS (at $90 \%$ ) and of ERL (at 95\%) in the X-ray band.

The most interesting aspect is the extension to a factor 100 lower fluxes provided by the X-ray selection of the ERL. Indeed assuming typical $\alpha_{\mathrm{rx}}(\sim 0.8),\left\langle\alpha_{\mathrm{r}}\right\rangle$ and $\left\langle\alpha_{\mathrm{x}}\right\rangle$, an X-ray flux level of $\sim 10^{-12} \mathrm{erg} \mathrm{cm}^{-2} \mathrm{~s}^{-1}$ corresponds to $\sim 0.3 \mathrm{Jy}$ in the radio band (both for FS and SS). At these levels, the counts of FS exceed the prediction of the beaming model proposed by PU92. Furthermore if one extrapolates to lower radio fluxes (say by a factor $\sim 10$ as in the $\mathrm{X}$-ray band), the radio counts of $\mathrm{X}$-ray selected objects are marginally consistent with the Euclidean extrapolation from higher fluxes and thus largely exceed (factor $>10$ for FS and $\sim 3$ for SS) those predicted by the beaming model, which drop below $\sim 0.1 \mathrm{Jy}$. Note that because of the X-ray threshold the radio counts of ERL can be only a lower limit to the actual surface density of radio-loud quasars.

It is also relevant to compare the ERL counts with those of BL Lacs extracted from the EMSS. As shown in Fig. 7, the BL Lac distribution is flatter, dominat-

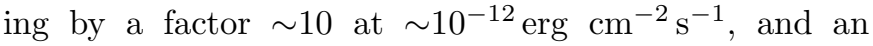
"inversion" of the two populations occurs just above $\sim 310^{-13} \mathrm{erg} \mathrm{cm}^{-2} \mathrm{~s}^{-1}$. Therefore while at high X-ray fluxes RL quasars are as rare, or rarer, than BL Lacs, deep, small area surveys are bound to find large numbers of them.

precisely we obtain: FS: $0.32 \pm 0.36(0.15 \pm 0.34)$, SS: $0.96 \pm$ $0.14(0.85 \pm 0.20)$, for a dividing $\alpha_{\mathrm{r}}=0.7(0.5)$. 


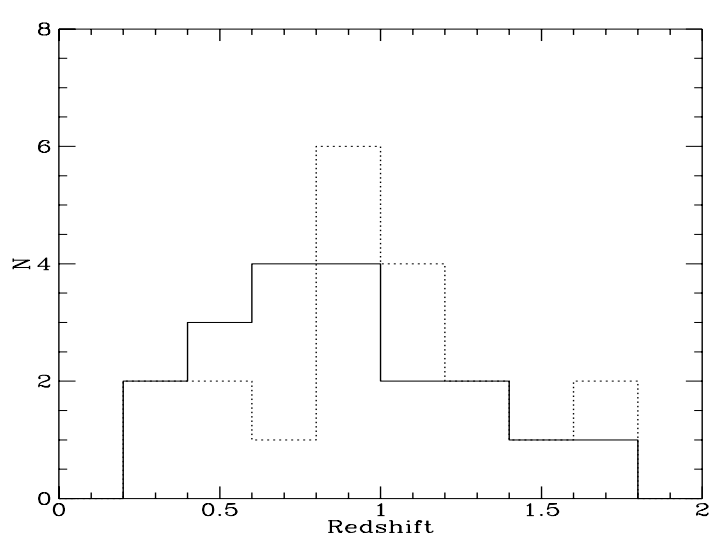

Fig. 8. Redshift distribution for SS sources (solid line) and FS sources (dotted line)

\subsection{Redshift distribution}

All sources are below redshift of $\sim 2$, with a broad peak around $z \sim 1$. This is due to the sky coverage of the EMSS, combined with the LF of the AGN. There is only marginal evidence for SS to be at redshifts lower than FS (See Table 2. Further, the probability of getting by chance a larger $\mathrm{KS}$ value is $19.6 \%$ ).

The typical $z$ are globally similar (within $1 \sigma$ ) to the average redshifts of FSRQ and SSQ selected in the radio band (2 Jy), while they are significantly larger than those of FR II radio galaxies, as already noted by PU92.

\section{3. $V_{\mathrm{e}} / V_{\mathrm{a}}$}

In order to quantify the evolutionary properties of the sample, we estimated, again subdividing it into FS and $\mathrm{SS}$, the $V_{\mathrm{e}} / V_{\mathrm{a}}$ parameter (Avni \& Bachall 1980), a generalization of the $V / V_{\max }$ statistics (Schmidt 1968) when complete samples at different flux limits are combined. The results are shown in Table 2. Positive evolution is required for the subsamples and the whole population at $>4 \sigma$ level, while no statistical difference can be found between the FS and SS behavior.

A systematically lower $V_{\mathrm{e}} / V_{\mathrm{a}}$ (though consistent within $\sim 2 \sigma$ ) is found for the quasars of the 2 Jy sample (PU92), possibly indicating a tendency of faster evolution of the X-ray selected objects ${ }^{4}$.

In order to compare the results with those relative to the 2Jy objects, we characterize the evolution in terms of an exponential pure luminosity function of the form $L(z)=L_{(z=0)} \mathrm{e}^{-C t}$, where $1 / C$ is the look-back time in units of the Hubble time. Note however that for the whole AGN sample in the EMSS, this form was found to be adequate only if a luminosity dependent value for $C$ was introduced (Della Ceca et al. 1992). The derived evolution rate is consistent (at $\sim 2 \sigma$, see Table 2 ) with that inferred for FSRQ (and once again it is indistinguishable between

\footnotetext{
${ }^{4}$ As this behavior is opposite to that of BL Lacs derived from the EMSS, this indirectly (re)confirms the reality of the uncommon negative evolution found for XBL.
}

FS and SS). As our sample is limited to $z<2$ nothing can be deduced on a possible decline in the number density of sources at high redshifts (e.g. Dunlop \& Peacock 1990).

Also a power law luminosity evolution $L(z)=$ $L_{(z=0)}(1+z)^{\gamma}$ can well represent the cosmological changes. The required dependence on $z$ is similar to what is found for radio-quiet objects (Table 2). The results for the FS sources are again consistent with the whole EMSS AGN behavior, while SS require a slightly higher value of $\gamma$ (at $1 \sigma)$.

For computing fluxes and luminosities we have assumed that $\alpha_{\mathrm{x}}=1$ - supported by the fact that $\left\langle\alpha_{\mathrm{x}}\right\rangle \sim 1$ in the ERL sample and by a study of the IPC Hardness Ratio for the EMSS AGN (Maccacaro et al. 1988). Note however that for X-ray spectra with a slope different from 1 the effect of the cosmological evolution has the same functional form of the K-correction factor and, in first approximation, the best-fit value of $\gamma$ is lower (if $\alpha_{\mathrm{x}}>1$ ) or higher (if $\alpha_{\mathrm{x}}<1$ ) by an amount $\sim\left|1-\alpha_{\mathrm{x}}\right|\left(\right.$ for $\left|1-\alpha_{\mathrm{x}}\right|<1$ ).

\subsection{Luminosity function}

Finally, we built the luminosity functions (LF) of FS and SS, both as observed and applying the two evolutionary models to de-evolve luminosities at $z=0$ (Figs. 9a,b). As expected, no difference is found between flat and steep spectrum objects (the extension of FS a factor of $\sim 10$ more in luminosity is due to only one source). The distributions have been fitted with power-laws (see results in Table 2), as the large uncertainties do not allow to better characterize their shape.

At odds with these results, a significant difference in the number density of flat and steep spectrum objects is found in radio selected samples (2 Jy), with FSRQ numerically dominating above $\sim 10^{43} \mathrm{erg} \mathrm{s}^{-1}$. Converted into the $\mathrm{X}$-ray band, this luminosity corresponds to $\sim 10^{45} \mathrm{erg} \mathrm{s}^{-1}$, where however a smaller number of SS, within the volume explored, are found.

The slope of the local LF of ERL is significantly steeper than that of FSRQ, revealing a first clear difference between the population of radio selected and X-ray selected flat spectrum quasars. This discrepancy is of course present also with respect to the X-ray LF computed by Maraschi \& Rovetti (1994) from the radio LF of FSRQ and SSQ over the common range in X-ray luminosity.

The total number densities of FSRQ is predicted to be $\sim 2.2 \mathrm{Gpc}^{-3}$ above $L_{\mathrm{x}} \sim 510^{43} \mathrm{erg} \mathrm{s}^{-1}$ (PU92) with a parent population of $81 \mathrm{FR}$ II $\mathrm{Gpc}^{-3}$. Locally we find twice this density. Also, SS are $\sim 7$ times those predicted by extrapolating below the cutoff the PU92's model predictions: in other words we do not find evidence for either a cut off or a flattening of the LF below X-ray powers of $\sim 310^{44} \mathrm{erg} \mathrm{s}^{-1}$. XBL have a slope slightly flatter than the ERL one and a local number density (at say $10^{44} \mathrm{erg} \mathrm{s}^{-1}$ ) about 50 times larger. 

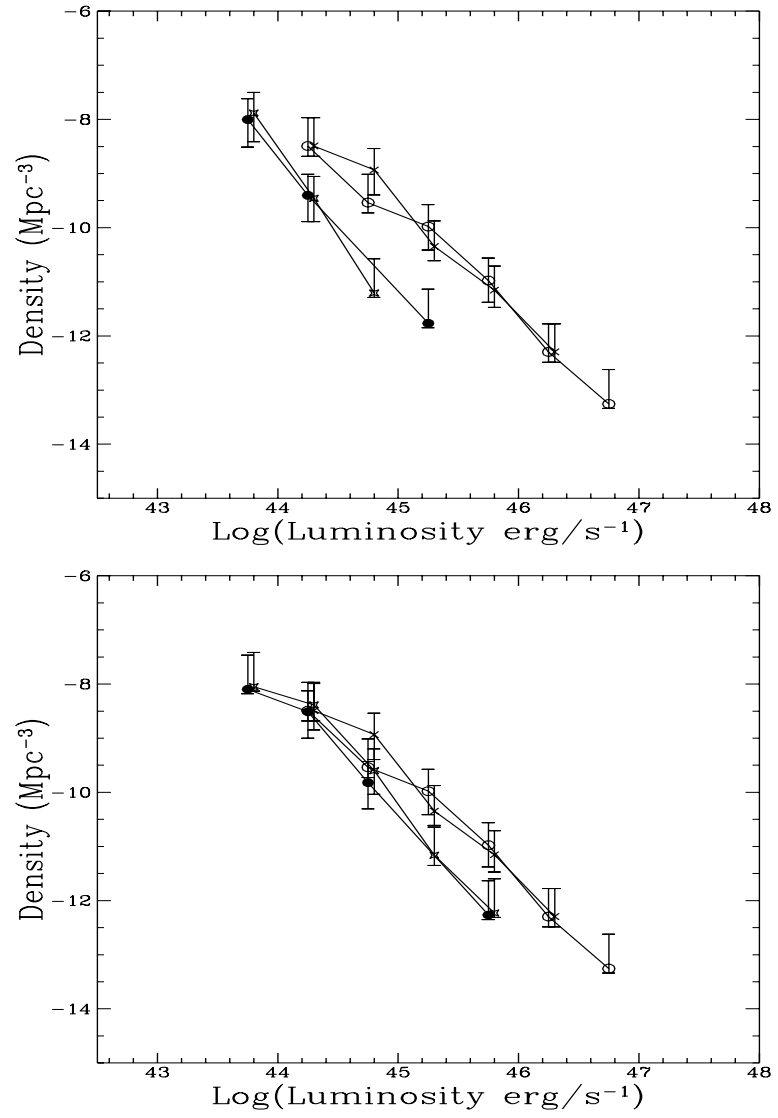

Fig. 9. Differential luminosity functions for the SS and FS sources, in step of $0.5 \log L$ (symbols as in previous figures; filled circles and stars indicate de-evolved SS and FS sources respectively). The SS points have been slightly shifted artificially on the luminosity axis for clarity. The de-evolving form is: upper panel exponential, lower panel power law

Also the ratio of FS/SS found in the EMSS significantly differ from that estimated by Maraschi \& Rovetti (1994), i.e. a ratio of 30 between the two populations, while at the corresponding X-ray luminosity we find similar numbers of FS and SS. In particular the density of FS is about one tenth than their predictions ${ }^{5}$.

The same "excess" of SS with respect to FS in the $\mathrm{X}$-ray selected sample clearly reflects in the relative ratio of SS/FS $=19 / 20$ found in the EMSS, compared to $34 / 50$ found in radio selected survey at higher flux levels (2 Jy) - despite SS do not dominate at low radio luminosities. This seem to imply that the radio selection is more biased than the X-ray one in finding FSRQ with respect to SSQ.

\subsection{Predictions: Number counts and redshift distributions}

Finally, let us consider the number counts and redshift distributions predicted by the above findings. In Fig. 10a the

\footnotetext{
${ }^{5}$ Note that for a flat spectral index $\alpha_{\mathrm{r}} \sim 0$ the threshold flux would be comparable at higher selection radio frequencies, therefore implying that the discrepancy is likely not due to the (radio) selection band.
}
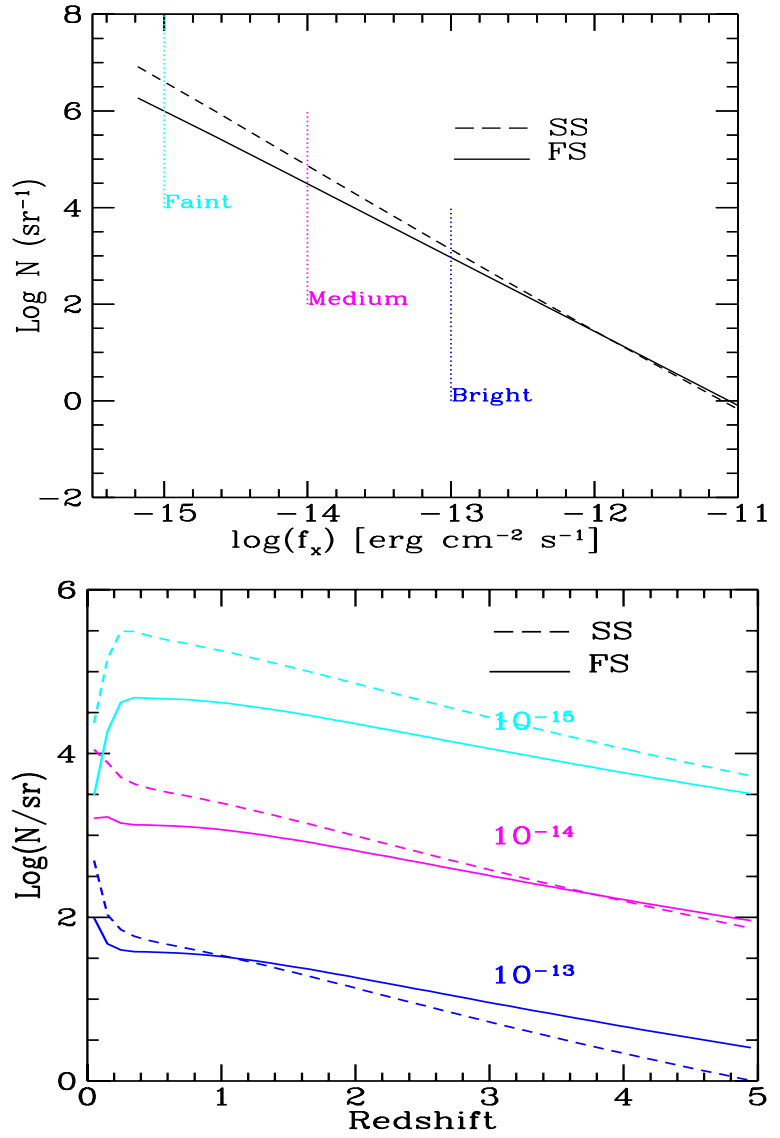

Fig. 10. a) Predicted number counts for the FS (solid line) and SS (dashed line) at flux levels reachable by Chandra and Newton-XMM, in the soft band, for a population extending up to $z_{\max } \sim 5$. b) Redshift distributions of FS and SS, derived from the evolving LF, at different flux limits

number counts extrapolated at flux levels observable by Chandra and Newton-XMM are reported. These have been derived by the integration of the evolving LF (of FS and SS) up to redshift $z_{\max } \sim 5$ (between $L_{\mathrm{x}}=10^{41}-10^{47} \mathrm{erg}$ $\mathrm{s}^{-1}$ ). The limits of the proposed Newton-XMM surveys are plotted in Fig. 10a. Given the areas that will be probably covered at each flux limit $(\sim 1,5$, and 150 sq. deg. respectively in the faint, medium and bright survey), we expect about 2000 (300), 150 (50), 100 (45) SS (FS) quasars in each, if the measured evolution is taken at face value and the extrapolation holds at the faint fluxes. These samples will be large enough to allow refined prediction on the radio-loud quasars properties. The corresponding redshift distributions at different flux limits are shown in Fig. 10b, where SS can be seen to dominate at lower $z$ at all flux thresholds.

\section{Summary and discussion}

We have constructed the first complete X-ray selected sample of radio-loud quasars. This has allowed us to investigate both the spectral properties and possible trends among blazars and the statistics of such sources in the frame of unification models for radio-loud objects. 
Through X-ray selection (at the level of $\sim 10^{-13} \mathrm{erg} \mathrm{cm}^{-1} \mathrm{~s}^{-1}$ ) we explore the same range of redshifts and $\mathrm{X}$-ray and optical luminosities of radioselected objects, but we find quasars significantly fainter in the radio band.

As far as the spectral properties of blazars are concerned, FS ERL do not differ significantly from radio selected sources. However, the decrease in radio luminosity correlates with the increasing frequency of the spectral peak, as indicated by the trend of the broad band spectral indices. This supports the existence of a sequence in the blazar family, where the peak decreases in energy with increasing source radio power (Fossati et al. 1998; Ghisellini et al. 1998).

Furthermore, although ERL are selected at X-ray luminosities comparable to those of HBL, we find no evidence for the existence of quasars whose synchrotron emission component peaks at $\mathrm{X}$-ray frequencies. This is also indicated by the similarity of $\alpha_{\mathrm{rx}}$ of ERL and FSRQ, which suggests that the synchrotron component does not dominate the $\mathrm{X}$-ray band luminosity (see e.g. Fossati et al. 1998; see also Padovani 2001).

On one hand in any unifying scenario some overlapping between objects of different characteristics might be naturally expected (likely corresponding to nuclei changing character or mismatching the extended radio morphologies). On the other hand the ERL spectral distributions appear to strongly resemble those of LBL over a large range of powers ( $L_{\mathrm{r}}$ spans three decades), where both broad lined and lineless objects $\mathrm{co}^{-}$-exist. This suggests that the line typology might not be univocally related to the source power and broad band spectral shape. Indeed, one might envisage that the (occasional) appearance of broad line components in BL Lacs is related to a change occurring in the nuclear source - but at such low luminosity levels that the lines never become quasar-like - while the blazar sequence of power vs continuum spectral behavior would be a separate effect and the resemblance of the broad band spectral indices of LBL and ERL might be indeed attributed to a similar trend of the synchrotron peak position with (radio) luminosity.

However, from the paucity of information we have on ERL, this similarity can be due to the progressive dominance of a broad thermal component (blue bump or contribution from the host galaxy in FS and SS, respectively) over the non-thermal emission in objects of lower nonthermal radio luminosity. Accounting for this effect in fact reduces the intrinsic overlapping of the two populations: decreasing the optical luminosity by considering only the (actual) non-thermal emission corresponds to move ERL towards FSRQ in the spectral index plane. If so, the tighter relation between source power and SED shape (and line strength) would be re-enforced by the ERL sample. Clearly this ambiguity can be solved by the estimate of the optical and X-ray spectra of ERL. Support to this view also comes from the findings of the REX survey (Caccianiga et al. 2000), where a significant number of sources are found with clear optical excesses with respect to FSRQ and LBL (see their Fig. 6), while the radio and $\mathrm{X}$-ray luminosities are simply linearly correlated.

We stress here that also the strong difference in the statistical properties of lineless and lined samples indicates that the two populations are not simply analogous manifestations of the same phenomenon, where only the line intensities are different.

We find no indication of any dependence of the spectral properties on redshift, nor any difference between the nuclear properties of flat and steep spectrum radio quasars, when selected in the $\mathrm{X}$-ray band, except possibly flatter counts for the FS. In other words the nuclear component seems not to be directly dependent on the extended (radio) properties.

From the statistical point of view, the radio and $\mathrm{X}$-ray selection sample the same population at the brightest fluxes. The main new information we obtain is that at the lower flux levels sampled through the $\mathrm{X}$-ray selection the radio source counts do not drop - and not even flatten - as predicted by some beaming models (e.g. PU92). Surveys at low radio flux levels $(\sim \mathrm{mJy})$ will be able to establish the presence or absence of such a decline in the population.

The ERL sample has provided also with the possibility of directly comparing the X-ray evolution of quasars with that of BL Lacs. This is particularly relevant both for understanding the reason of the negative evolution of BL Lacs in this band and in particular as a direct test of models postulating a physical evolution of radio-loud quasars into BL Lacs (e.g. Cavaliere \& Malquori 1999).

The ERL population significantly evolves, at a rate indistinguishable from that of FSRQ. With respect to the radio selection, the $\mathrm{X}$-ray one provides also with a different number ratio of flat and steep radio sources, with the latter comparable to the former ones. This number ratio does not appear to depend on the radio luminosity, but significantly depends on the radio - and possibly on the $\mathrm{X}$-ray - flux, being the X-ray selection less biased at low radio fluxes. Both deeper radio surveys and the definition and study of optically selected blazars - as that which will be provided by the EIS surveys (e.g. Cagnoni \& Celotti 2001) - should confirm this trend.

Due to the dramatic effect of beaming in altering the shape of the luminosity functions, it is not possible to simply use the above ratio as an indicator of the beaming angles subtended by FS and SS. Recent indications of the existence of a slower moving "layer" jet component dominating the observed emission in radio galaxies (e.g. Laing 1993; Chiaberge et al. 2000) further modify the expected number ratio of beamed and parent population sources. Furthermore as the luminosity functions do not show a turn over at low powers it is not even possible to provide model independent estimates on the total number of objects of the two populations. Nevertheless it is clear that the beaming parameters and number density of the parent and beamed populations have to be revised in the light of the new and less biased information provided by the ERL, affecting the predictions on the radio-activity in AGN and 
galaxies as well as the contribution of these objects to the $\gamma$-ray background.

\section{Appendix A: Sample selection: Notes on individual objects}

Two of the 43 radio-loud objects of the EMSS have been classified as Narrow Emission Line objects (MS 1252.40457 as a Seyfert 2 galaxy, and MS 1414.8-1247 as an HII region; Boyle et al. 1995) and therefore we remove them from the sample under study.

MS $0234.8+0655$ is detected at $6 \mathrm{~cm}$, but not at $20 \mathrm{~cm}$. By using the EMSS radio flux the source is radio-loud $\left(\alpha_{\text {ro }}=0.43\right)$, but with the NVSS flux limit $(=2.5 \mathrm{mJy}) \alpha_{\mathrm{r}} \leq-0.81$. Therefore both MS 0234.8+0655 and MS 2134.0+0028 $\left(\alpha_{\mathrm{r}}=-0.88\right)$ have a highly inverted spectrum and are removed from the sample.

We examined also the case of MS 1253.6-0539 that has a very high upper limit from the EMSS data (5.5 mJy at $5 \mathrm{GHz}$ ) and therefore in principle could be a radio source. However, the object is also not detected at $1.4 \mathrm{GHz}$ by the NVSS at a limit of $2.5 \mathrm{mJy}$. Furthermore, it appears in Table 10 of Stocke et al. (1991) as having an ambiguous spectrum due to the poor signal to noise. We therefore do not include it in the sample.

Note that MS 0833.3+6523 (a.k.a. 3C 204) has $F_{\text {core }}^{6 \mathrm{~cm}} \sim$ $30 \mathrm{mJy}$ (from the EMSS VLA data) while $F_{\text {tot }}=369 \mathrm{mJy}$ : the extended flux dominates the emission and we use the latter to compute the radio spectral index. The same applies to MS $1623.4+2712$, for which the EMSS $F_{\text {core }}^{6 \mathrm{~cm}}=$ $43 \mathrm{mJy}$ while $F_{\text {tot }}=240 \mathrm{mJy}$.

Acknowledgements. We thank Alessandro Caccianiga, Roberto Della Ceca and Laura Maraschi for useful comments and discussions. The Italian MURST is acknowledged for financial support - partly under the Program Cofinanziamento 1999.

\section{References}

Avni, Y., \& Bachall, J. N. 1980, ApJ, 235, 694

Becker, R. H., White, R. L., \& Edwards, A. L. 1991, ApJS, 75,1

Boyle, B. J., McMahon, R. G., Wilkes, B. J., \& Elvis, M. 1995,
MNRAS, 276, 315

Brinkmann, W., Yuan, W., \& Siebert, J. 1997, A\&A, 319, 413

Caccianiga, A., Maccacaro, T., Wolter, A., Della Ceca, R., \& Gioia, I. M. 2000, A\&AS, 144, 247

Cagnoni, I., \& Celotti, A. 2001, Mem. S. A. It, in press

Cavaliere, A., \& Malquori, D. 1999, ApJ, 516, L9

Celotti, A., Maraschi, L., Ghisellini, G., Caccianiga, A., \& Maccacaro, T. 1993, ApJ, 416, 118

Chiaberge, M., Celotti, A., Capetti, A., \& Ghisellini, G. 2000, A\&A, 358, 104

Condon, J. J., et al. 1998, AJ, 115, 1693

Della Ceca, R., Maccacaro, T., Gioia, I. M., Wolter, A., \& Stocke, J. T. 1992, ApJ, 389, 491

Della Ceca, R., et al. 1994, ApJ, 430, 533

Dunlop, J. S., \& Peacock, J. A. 1990, MNRAS, 247, 19

Fossati, G., Maraschi, L., Celotti, A., Comastri, A., \& Ghisellini, G. 1998, MNRAS, 299, 433

Ghisellini, G., \& Maraschi, L. 1986, ApJ, 340, 181

Ghisellini, G., Celotti, A., Fossati, G., Maraschi, L., \& Comastri, A. 1998, MNRAS, 301, 451

Gioia, I. M., et al. 1990, ApJS, 72, 567

Gregory, P. C., \& Condon, J. J. 1991, ApJS, 75, 1011

Kendall, M., \& Stuart, A. 1979, The Advanced Theory of Statistics (McMillan, New York)

Laing, R. A. 1993, in Space telescope Sci. Inst. Symp. 6: Astrophysical Jets, ed. D. Burgarella, M. Livio, \& C. P. O'Dea (Cambridge University Press, Cambridge), 95

Laurent-Muehleisen, S. A., Kollgaard, R. I., Feigelson, E. D., Brinkmann, W., \& Siebert, J. 1999, ApJ, 525, 127

Maccacaro, T., Gioia, I. M., Wolter, A., Zamorani, G., \& Stocke, J. T. 1988, ApJ, 326, 680

Maccacaro, T., et al. 1994, Astrophys. Lett. Comm., 29, 267

Maraschi, L., \& Rovetti, F. 1994, ApJ, 436, 79

Morris, S. L., et al. 1991, ApJ, 380, 49

Padovani, P. 2001, in Blazar Physics and Demographics, Baltimore, July 2000, in press

Padovani, P., \& Giommi, P. 1995, ApJ, 444, 567

Padovani, P., \& Urry, M. C. 1992, ApJ, 387, 449 (PU92)

Perlman, E. S., et al. 1998, AJ, 115, 1253

Schmidt, M. 1968, ApJ, 151, 393

Stark, A. A., et al. 1992, ApJS, 79, 77

Stocke, J. T., et al. 1985, ApJ, 298, 619

Stocke, J. T., et al. 1991, ApJS, 76, 813

Urry C. M., \& Padovani, P. 1995, PASP, 107, 803

Wall, J. V., \& Peacock, J. A. 1985, MNRAS, 216, 173

Wolter, A., Gioia, I. M., Maccacaro, T., Morris, S. L., \& Stocke, J. T. 1991, ApJ, 369, 314 